\section{Phonological awareness intervention for verbal working memory skills in school-age children with specific language impairment and concomitant word reading difficulties}

Park, Jungjun $\square$

Baylor University, United States (Jungjun_park@baylor.edu)

Ritter, Michaela

Baylor University, United States (Michaela_ritter@baylor.edu)

Lombardino, Linda

University of Florida, United States (llombard@coe.ufl.edu)

Wiseheart, Rebecca

St. John's University, United States (wisehear@stjohns.edu)

Sherman, Susan

Baylor University, United States (Susan_sherman@baylor.edu)

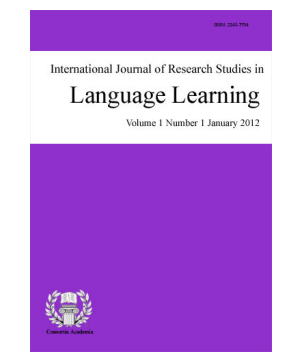

ISSN: 2243-7754 Online ISSN: 2243-7762

OPEN ACCESS

$\begin{array}{lll}\text { Received: } 01 \text { August } 2013 & \text { Revised: } 14 \text { October } 2013 & \text { Accepted: } 15 \text { October } 2013\end{array}$ Available Online: 29 November $2013 \quad$ DOI: $10.5861 /$ ijrsll.2013.534

\title{
Abstract
}

This exploratory investigation examined the effects of explicit phonological awareness intervention on each subcomponent of Baddeley's verbal working memory model. Fifty school-age children with specific language impairment (SLI) and concurrent deficits in word reading were randomly assigned to either an experimental $(n=25)$ or a control group $(n=25)$. Children in both groups received individual traditional language intervention for four, 1 hour sessions each week for 4 weeks (16 hrs). The experimental group received an additional 20 min of phonological awareness intervention each day $(5.3 \mathrm{hrs})$. Participants in the experimental group significantly outperformed the children in the control group across all verbal working memory measures. The strongest effects were found for the digit recall and word list recall subtests, which were used to assess the verbal short-term memory component of the model (i.e., phonological loop). The next largest effect sizes were reported for the subtests of the verbal working memory functioning (i.e., phonological loop and central executive). The smallest change was found on the recalling sentences subtest, which was chosen to represent the episodic buffer component. These results suggest that school-age children with SLI and concomitant word-reading difficulties in second through third grade who receive explicit phonological awareness intervention can make significant gains on untrained verbal working memory skills in a relatively short period of time which underscores the importance of phonological awareness intervention beyond first grade.

Keywords: specific language impairment (SLI); reading deficit; phonological loop; central executive; episodic buffer; school-age children 


\section{Phonological awareness intervention for verbal working memory skills in school-age children with specific language impairment and concomitant word reading difficulties}

\section{Introduction}

Children with specific language impairment (SLI) are characterized as having difficulty developing age-appropriate language skills in the absence of hearing, neurological, emotional, or intellectual impairment (Leonard, 1998). Verbal working memory is the ability to retain verbal information in temporary storage while processing other cognitively demanding activities for higher linguistic functions (Baddeley, 1986, 2000). Over the past two decades researchers have established a strong relationship between language learning difficulties in children with SLI and verbal working memory (Archibald \& Joanisse, 2009; Dollaghan \& Campbell, 1998; Gathercole \& Baddeley, 1990). Specifically, there is a considerable amount of research that cites variations in verbal working memory capacity as an underlying cause of individual differences in a myriad of language skills, including vocabulary acquisition (Weismer \& Edwards, 2006), syntactic knowledge (Swanson \& Berninger, 1996), sentence comprehension (Montgomery, 2000), and listening comprehension (King \& Just, 1991; Montgomery \& Evans, 2009). It is argued that "the striking deficits of children with specific language impairment in these two key domains (i.e., verbal short-term memory and verbal working memory) make a major contribution to the learning difficulties experienced by these children" (Archibald \& Gathercole, 2006, p. 154).

Moreover, close links are well established between SLI and word-level reading difficulties, which are causally related to phonological processing deficits (Catts, Adlof, Hogan, \& Weismer, 2005; Catts, Bridges, Little, \& Tomblin, 2008). Even though not all children with SLI manifest deficits in word reading, it is reported that as many as one third of the children diagnosed with SLI in kindergarten, are found to have phonologically based word reading deficits (i.e., dyslexia) during the school years (Catts et al., 2005). In other studies, the comorbidity between SLI and word reading deficits during school years is reported to be approximately $40 \%$ (Snowling, Bishop, \& Stothard, 2000) to 50\% (McArthur, Hogben, Edwards, Heath, \& Mengler, 2000).

Furthermore, children with SLI who had comorbid word reading deficits have been reported to have problems in verbal memory (Catts et al., 2005). There is now substantial evidence that poor word decoding skills are highly associated with impairment in verbal working memory (e.g., Roodenrys \& Stokes, 2001; Swanson \& Ashbaker, 2000). In fact, verbal working memory is a crucial component of phonemic sequencing and blending skills, which are necessary for decoding unfamiliar words or pseudo-words (Troia, Roth, \& Yeni-Komshian, 1996). For successful word decoding, young readers have to decode a series of graphemes, and temporarily hold the phonemes in a phonological storage in order to carry out the cognitively extensive manipulation task of blending. Thus, these findings are not surprising because efficient phonetic recoding from orthographic input would undoubtedly place high demands on verbal working memory in general and verbal short-term memory in particular.

On the other hand, it was suggested that weak and less-specific phonological representation of words stored in long-term, lexical memory is an underlying cause of verbal working memory skills manifested by children with SLI (Bishop \& Snowling, 2004; Pennington \& Bishop, 2009; Roodenrys, Hulme, \& Brown, 1993; Shankweiler, 1989). Henry and Millar (1993) suggest that the development of verbal working memory capacity is a direct result of the quality or specification of underlying phonological representations. That is, words with well-specified phonological representations are easier to retrieve because they are less vulnerable to degradation and easier to reconstruct or "reintegrate" in verbal working memory (Fowler, 1991). There is a vast amount of research to suggest that explicit phonological awareness training emphasizing the sound structure of words can facilitate the child's understanding of the segmental structure of speech. Therefore, we can anticipate that improvement in the quality and accessibility of phonological representations through phonological awareness 
training (Rvachew, Nowak \& Cloutier, 2004) can enhance performance on verbal working memory tasks (Melby-Lervåg \& Hulme, 2010), which may lead to improvement in other areas of language learning difficulties experienced by children with SLI.

This current study was conducted to explore whether explicit training in phonological awareness can generalize to the distinct functions of the verbal working memory system proposed by Baddeley and Hitch (1974) and Baddeley (1986, 2000, 2003): verbal short-term memory, verbal working memory, and the episodic buffer. Specifically, we sought to explore to what extent each of these verbal memory functions could be improved in a subgroup of school-age children with SLI and concurrent deficits in word reading and verbal working memory through a phonological awareness intervention program, which was originally designed to foster phonemic awareness and word decoding skills.

\subsection{Baddeley's verbal working memory model}

Verbal working memory is the ability to retain verbal information in temporary storage while processing other cognitively demanding activities for higher linguistic functions (Baddeley, 1986, 2000). Arguably, the most widely cited model is the one proposed by Baddeley and Hitch (1974) and subsequently updated by Baddeley $(1986,2000,2003)$, which now includes three substrates of verbal working memory: the phonological loop (PL), the central executive (CE), and the episodic buffer (EB). The PL serves as temporary storage of small amounts of information for a short period of time and it has two subcomponents: the phonological store and subvocal rehearsal. The PL is typically assessed by simple span tasks that need only temporary retention of verbal material such as nonword repetition or simple digit recall. According to Baddeley's framework, verbal short-term memory performance is mediated by the PL. The CE is a limited capacity component which is responsible for control of attention, allocating memory resources, and regulation of the flow of information into working memory.

Verbal working memory tasks that impose simultaneous demands on storage and processing capacity rely both on the domain-specific store and the central executive to support processing (i.e., PL + CE). A good example of a verbal working memory test is listening span task, in which participants are asked to verify the semantic appropriateness of a set of spoken sentences and then recall the last words of each sentence in the same order they were presented. To properly perform on this task, one should have a high degree of executive attentional-control and temporary storage (Conway, Kane, \& Engle, 2003; Kane et al., 2007). The collateral processing needed to perform complex working memory tasks requires divided attention, allocation of multiple mental resources, and active maintenance of conscious attention, which fall under the purview of the central executive (Halpern, McMillan, Moore, Dennis, \& Grossman, 2003).

The third component is the episodic buffer, which is envisaged as a temporary storage facility that integrates information from multiple sources (e.g., long term memory) into a single episode (Baddeley, 2000; Baddeley \& Wilson, 2002). For example, tasks involving verbatim sentence repetition have been identified as reliable measures of the episodic buffer. Baddelely proposed that the episodic buffer is critically important for sentence recalling tasks because tasks of this nature are highly constrained not only by the formulation of phonological representations in verbal short-term memory (i.e., phonological loop), but also by the accessibility to syntactic structures and semantic representations of words in long-term memory. For the current study, the term verbal memory is used to refer to three functional aspects of Baddeley's verbal working memory model, including verbal short-term memory (i.e., PL), verbal working memory (i.e., PL + CE), and episodic buffer (EB) functions.

\subsection{Common operational demands of phonological awareness and verbal working memory}

Phonological awareness refers to the ability to think about, identify, and manipulate the various components of words such as syllables, onset/rimes, and phonemes (Griffith \& Olson, 1992, Chou, Wang, \& Ching, 2012). Poor phonological awareness can contribute to difficulties translating orthography to phonology resulting in 
problems with fluent word decoding, which negatively impacts reading comprehension and fluency (Catts, Fey, Zhang, \& Tomblin, 2001; Lyon, Shaywitz, \& Shaywitz, 2003, Vitale \& Nancy, 2012). Considering that phonological awareness is a strong predictor of word decoding and is fundamental to the reading process (e.g., Hurford \& Sanders, 1990), it is not surprising that children with SLI and poor phonological awareness skills are as much as six times more likely than children with typical language development to have difficulties with learning to read (Catts, Fey, Tomblin, \& Zhang, 2002).

Close links are well established between children's verbal working memory and phonological awareness skills. Schuele and Boudreau (2008) argued that the phonological loop is required for all phonological awareness tasks regardless of the operational complexity. Similarly, the operational efficiency of phonological awareness is determined by the integrity of the verbal working memory system (Castles \& Coltheart 2004). For instance, in order to complete a rime oddity task, not only is the child required to hold the phonological representations of each word in short-term store, but also he has to maintain the phonological codes long enough to compare the rime parts of multiple stimuli words. Oakhill and Kyle (2000) have found that central executive function plays an important role in phonological awareness tasks as well (e.g., the rhyme oddity task). Navarro et al. (2011) also found a strong correlation between central executive and phonological awareness tasks in a group of 424 typically developing children (age: 4 to 7 years). Thus, successful phonemic deletion is also contingent upon the capacity of verbal working memory to store the target word and locate the phoneme to be deleted. These findings can be explained in terms of the function of executive processing, which acts to distribute appropriate attentional resources to the phonological loop for particular processes required for phonological awareness tasks.

In support of this interplay between phonological awareness and verbal working memory, a number of studies have demonstrated a strong positive correlation between these two constructs (Siegel \& Linder, 1984; Stanovich, Cunningham, \& Freeman, 1984). Researchers have interpreted this correlation as reflecting a common phonological processing substrate between the two constructs (e.g., De Jong, Seveke, \& van Veen, 2000; Garlock, Walley, \& Metsala, 2001; Leather \& Henry, 1994; Oakhill \& Kyle, 2000). Along with correlational data on behavioral measures, recent neuroimaging studies have substantiated the involvement of verbal working memory in various phonological awareness tasks. The neural substrates underlying both verbal working memory and phonological awareness tasks appear to be located in overlapping brain regions (Shaywitz \& Shaywitz, 2005). In the same vein, Kovelman et al. (2012) reported that the left dorsolateral prefrontal cortex, a brain region known to subserve verbal working memory tasks, was also highly activated when typical readers were engaged in a rime detection task.

Given the operational interconnectedness of phonological awareness and verbal working memory skills, it seems plausible that phonological awareness training could be used as a viable treatment option to improve poor verbal working memory skills of children with SLI on the basis that mental manipulation of sound units of words requires "practice" of the same collateral processes involved in mental manipulation of verbal materials required for verbal working memory tasks.

\subsection{Phonological awareness training as a tool of verbal working memory improvement}

Unfortunately, only a small number of studies have investigated generalization effects between phonological awareness and verbal working memory intervention (Gillam \& van Kleeck, 1996; Schneider, Küspert, Roth, Vise, \& Marx, 1997; van Kleeck, Gillam, \& Hoffman, 2006). Gillam and van Kleeck (1996) conducted the first study in which 16 preschool aged children with speech and language disorders showed improvement both in phonemic awareness and verbal working memory following a phonemic awareness training program. Since then, however, only a handful of studies have been conducted that investigate the generalization effects of phonological awareness training on untrained verbal working memory components. Schneider et al. (1997) examined whether the effects of phonological awareness training generalize to other phonological processing skills such as verbal working memory in a cohort of normally developing children. After training 149 German kindergartners on a battery of phonological awareness skills for 6 months, children showed improved scores on PA, reading, and 
spelling tests at the end of first and second grade; however, no improvement was found on a word span task. In a more recent investigation, van Kleeck et al. (2006) reported contradictory findings. They provided phonological awareness training to 16 preschool children with SLI (mean age: 4 years 6 months) two times per week for 15 minute training sessions. Experimental training stimuli included various rhyming (e.g., identification and judgment) and phonemic awareness (e.g., sound identification/matching, phonemic blending/segmentation) activities. The participants' verbal working memory skills were measured on word and non-word span tasks and found to be significantly improved.

\subsection{Purpose of the current study}

To date, no intervention studies have been conducted to investigate the clinical efficacy of phonological awareness training on the verbal working memory skills of a group of school-age children with SLI and concomitant word reading difficulties beyond Grade 1. We implemented explicit phonological awareness intervention, which was originally designed to improve word decoding skills in a subgroup of school-age children (i.e., grade 2 to 3 ) who exhibited concurrent deficits in word-level decoding and verbal memory skills.

In light of the shared operational mechanism between phonological awareness and verbal working memory tasks, the first aim of this study was to determine if improvement in verbal memory skills could be achieved by explicit phonological awareness intervention in this subgroup of children. As discussed earlier, if the efficiency of phonological awareness and verbal working memory tasks are related on the basis of phonological representations and phonological awareness training strengthens underlying phonological representations, we can hypothesize that treatment gains will be reflected in improvements in verbal working memory skills.

Our second aim was to investigate the distinct effects of explicit phonological awareness intervention on the three substrates of verbal memory: verbal short-term memory, verbal working memory, and episodic buffer. As far as we know, no previous studies have been conducted on the efficacy of phonological awareness training on each of these three verbal memory components. As indicated by Schuele \& Boudreau (2008), we hypothesized that the potential gains would be most apparent on simple tasks assessing the capacity of verbal short-term memory function (i.e., PL) as the need to encode and hold the stimulus phonologically is required across all phonological awareness tasks. This hypothesis would be supported if phonological awareness intervention shows the strongest effects on the measures of phonological loop functions such as simple recall tasks (e.g., digit recall or word list recall). Relatively smaller clinical gains would be expected for tasks that are attention-demanding and employ both central executive and phonological storage (e.g., listening span, backward digit recall), and tasks that employ episodic buffer functions (e.g., sentences recalling).

\section{Method}

\subsection{Research design and procedure}

A pretest-posttest comparison group design was used to investigate the influence of phonological awareness intervention on the substrates of the verbal working memory system in school-age children with SLI, who manifested concurrent word decoding and verbal memory deficits. During the two weeks prior to the beginning of the intervention, both groups completed a set of assessments in verbal memory and word reading skills. Immediately after the conclusion of the four-week intervention period, participants' verbal memory and word reading skills were posttested. Pre- and post-intervention testing was conducted by four trained graduate assistants who were supervised by three certified speech-language pathologists. All testers remained blind to the participants' group membership. All tests were administered in a fixed order.

\subsection{Participant description}

Participants were recruited from a cohort of 91 children enrolled in a university-based speech-language 
Park, J., Ritter, M., Lombardino, L., Wiseheart, R., \& Sherman, S.

clinic located in the southwestern region of the United States. Of this cohort, 50 children attending second- and third-grade who met the inclusionary criteria participated in the study (30 boys and 20 girls). All participants had to have a diagnosis of SLI and demonstrate concurrent deficits in word-level reading and verbal memory tasks determined by an independent, certified speech-language pathologist. Of these 50 children, 25 children were randomly assigned to the experimental group which received both traditional language intervention and integrated phonological awareness intervention (i.e., PA-Tx Group). The remaining 25 children were assigned to a comparison group which received traditional language intervention only (i.e., LI-Tx Group).

A diagnosis of SLI for each participant was confirmed based on detailed review of the clinical records of each child. Children's language skills were assessed by the core language score of the Clinical Evaluation of Language Fundamentals, Fourth Edition (CELF-4; Semel, Wiig, \& Secord, 2003), which provided a summary of the participants' receptive and expressive skills. The core subtests consisted of Concepts and Following Directions, Formulating Sentences, Recalling Sentences, and, depending on the child's age, Word Knowledge (under 9 years) or Word Classes (9 years or older). A composite core language score was obtained for each child on the basis of the test norms. To qualify as having SLI, participants had to meet the following selection criteria: (1) a Core Language Score of $\leq 85$; (2) non-verbal IQ within normal range (i.e., $>85$ ) as measured by the Test of Nonverbal Intelligence - 4th Edition (TONI-4; Brown, Sherbenou, \& Johnsen, 2010); and (3) absence of a positive history for hearing loss, frank neurological damage, and emotional/psychological disability.

\section{Table 1}

Pre-intervention group performance and the results of t tests on the demographic characteristics, non-verbal intelligence, inclusionary criterion measures (spoken language and word-level reading), and verbal memory measures

\begin{tabular}{|c|c|c|c|c|c|c|}
\hline \multirow{2}{*}{ Measures } & \multicolumn{2}{|c|}{ PA-Tx } & \multicolumn{2}{|c|}{ LI-Tx } & \multirow{2}{*}{$\mathrm{t}(48)$} & \multirow[b]{2}{*}{$\mathrm{p}$} \\
\hline & M & SD & M & SD & & \\
\hline Gender(boys/girls)a & $14 / 11$ & & $16 / 9$ & & & \\
\hline Age (in months) & 92 & 12.94 & 95 & 13.71 & 1.15 & .25 \\
\hline TONI-4 & 99.36 & 9.09 & 101.2 & 6.96 & .43 & .66 \\
\hline \multicolumn{7}{|l|}{ Criterion measures } \\
\hline Spoken language (CELF-4 CLS) & 76.68 & 7.59 & 77.36 & 5.82 & .56 & .57 \\
\hline \multicolumn{7}{|l|}{ Word-level reading measures } \\
\hline Word Identification (WRMT-R) & 78.23 & 8.03 & 77.39 & 7.25 & .45 & .66 \\
\hline Word Attack (WRMT-R) & 76.86 & 7.88 & 78.74 & 5.99 & 1.02 & .31 \\
\hline \multicolumn{7}{|l|}{ Verbal memory measures } \\
\hline Digit Recallb & 79.80 & 9.56 & 81.24 & 9.22 & .69 & .49 \\
\hline Word List Recallb & 83.32 & 8.24 & 81.44 & 8.51 & .77 & .45 \\
\hline Listening Recallb & 79.32 & 11.0 & 82.48 & 7.99 & 1.27 & .21 \\
\hline Backward Digit Recallb & 81.60 & 10.6 & 79.48 & 12.5 & .88 & .39 \\
\hline Recalling Sentences (CELF-4) & 6.36 & 2.31 & 6.12 & 2.49 & .61 & .55 \\
\hline
\end{tabular}

Note. PA-Tx $=$ experimental group who received both traditional language therapy and additional PA intervention $(\mathrm{n}=25)$; LI-Tx $=$ age-matched control group who received only traditional language therapy $(\mathrm{n}=25)$; TONI-4 = Test of Nonverbal Intelligence - 4th Edition (TONI-4; Brown, Sherbenou, \& Johnsen, 2010); CELF-4 CLS = Core Language Scores of the Clinical Evaluation of Language Fundamentals-4 (Semel et al., 2003); WRMT-R = Woodcock Reading Mastery Test-Revised (Woodcock, 1988).

${ }^{\mathrm{a}}$ Group equivalence on gender was tested by chi-square analysis $[\chi 2(1, \mathrm{n}=50)=.725, p=.395]$. ${ }^{\mathrm{b}}$ Subtests of the Working Memory Test Battery for Children (WMTB-C; Pickering \& Gathercole, 2001).

The selection criteria for the word decoding deficits were a standard score of 85 or below on two single word reading subtests from the Woodcock Reading Mastery Test-Revised (WRMT-R; Woodcock, 1988): Word Identification and Word Attack. Participants' verbal memory abilities were assessed using five subtests from the Working Memory Test Battery for Children (WMTB-C; Pickering \& Gathercole, 2001) and the CELF-4. In order to be classified as having a verbal memory deficit, a child had to receive a standard score 85 or below on three of these five subtests. Table 1 displays participants' characteristics on the demographic and eligibility measures. The results of $t$ tests indicated that the two groups had equivalent performance at baseline for all criterion measures, including age, gender, nonverbal intelligence, spoken language, word reading, and verbal memory 
measures.

All participants passed a standard audiometric screening per American Speech-Hearing Association guidelines at the time of testing. All participants spoke English as their primary language (as reported by parents/caregivers). The ethnic backgrounds of the subjects were White (82\%), Hispanic/Latino (10\%), African American (4\%), and Asian American (4\%). Participants were not provided a monetary reward for this study and approval for all aspects of the present study including the recruitment letter, parental consent, and child consent forms was secured from the university's institutional review board. Children in the PA-Tx group ranged in age from 79 to 106 months $(M=92$ months, $S D=12.94)$. Children in the LI-Tx group ranged in age from 82 to 111 months $(M=95$ months, $S D=13.71)$.

\subsection{Outcome measures}

Verbal memory measures - Tests were chosen that tap skills representative of the verbal short-term memory, verbal working memory (phonological loop and central executive), and episodic buffer components of Baddeley (2000, 2003)'s models. Four subtests (i.e., Digit Recall, Word List Recall, Backwards Digit Recall, and Listening Recall) from the WMTB-C (Pickering \& Gathercole, 2001) and one subtest from the CELF-4 (i.e., Recalling Sentences) were administered. The WMTB-C is designed to assess working memory skills in children aged 5 through 15. The WMTB-C has been shown to be highly effective in child populations in distinguishing the phonological loop and central executive and has been validated against existing tests of achievement (Pickering \& Gathercole, 2001). The Recalling Sentences subtest from the CELF-4 was used to assess the episodic buffer function (Baddelely, 2000). The five verbal working memory tasks are described in detail below:

Verbal short-term memory. The Digit Recall and Word List Recall subtests from the WMTB-C (Pickering \& Gathercole, 2001) were used to assess verbal short-term memory (i.e., PL) functions. Both tasks require the encoding of phonological representations into temporary storage and the efficient recall supported by subvocal rehearsal and organizational attention of the materials in short-term memory. For the Digit Recall task, sequences of random digits are presented orally and the child is required to verbally repeat these digits verbatim. A maximum of six lists are presented at each length. For the Word List Recall subtest, a series of monosyllabic words composed of the consonant-vowel-consonant structure, are presented orally. The child is required to recall all the words in the list in the correct order.

Verbal working memory. The Backwards Digit Recall and Listening Recall subtests from the WMTB-C (Pickering \& Gathercole, 2001) were used to assess verbal working memory function. For the Backwards Digit Recall subtest, participants were required to recall a sequence of digits presented orally in the reverse order. For the Listening Recall task, the child first listens to a series of short declarative sentences, presented in blocks, and judge the semantic truthfulness of each sentence by stating "true" or "false" (e.g., "Oranges live in water" or "Roses smell nice."). The child then is asked to recall the last word of each sentence in the correct sequence immediately after the last sentence is presented (e.g., 'water', 'nice'). The number and length of sentences in each block are increased until the point at which the child can no longer accurately recall the last word.

Episodic buffer. As mentioned earlier, sentence repetition task requires the integration of verbal information from (Baddeley, 2003). In order to recall individual words that are sequenced to form a specific word order, the child has to integrate verbal information retrieved from temporary memory buffer with the outcomes of syntactic, morphological, and semantic analyses made the language processing system in the long-term memory. In this study, the Recalling Sentences subtest of the CELF-4 was used to assess the episodic buffer, where children are orally given a sentence and asked to repeat it verbatim. Sentences become increasingly more complex and longer. Responses are scored in relation to the number of errors made in each sentence.

Word-level reading - In order to assess the efficacy of our phonological awareness intervention for children with decreased word decoding skills, participants' word reading ability was posttested using the same pretest measures of the word reading of the WRMT-R (Woodcock, 1988). The Word Identification subtest assesses word 
Park, J., Ritter, M., Lombardino, L., Wiseheart, R., \& Sherman, S.

recognition for regularly and irregularly spelled words. The Word Attack subtest is used to decode phonetically regular nonwords. Children are asked to read increasingly complex words and nonwords in these tests.

\subsection{Intervention program}

As noted earlier, children were randomly assigned to either the PA-Tx or the LI-Tx group. Children in both groups received individualized traditional language intervention for four, two hour sessions each week for a 4-week period (i.e., a total of $16 \mathrm{hrs}$ of intervention). The PA-Tx group received an additional 20 min of phonological awareness intervention each day for four weeks (i.e., a total of $5.3 \mathrm{hrs}$ of intervention). The LI-Tx group did not receive this additional instruction. All intervention sessions were provided by trained graduate student clinicians under the supervision of three clinical supervisors.

Phonological awareness intervention - The phonological awareness intervention provided to the PA-Tx group was an adaptation of Goldsworthy's (2004) Phonological Awareness Training Program and was designed to foster phonemic awareness and word decoding skills. The phonological awareness intervention content was structured in terms of developmental complexity in line with findings from previous studies (Cunningham, 1990; Lundberg, Frost, \& Petersen, 1988). Specifically, the phonological awareness intervention progressed from phoneme identification/blending /segmenting to phoneme manipulation (deletion/replacement). The phonological awareness intervention included two sets of ten stimuli (e.g., words) that were provided for each individual objective. In order to progress to the next level of the phonological awareness intervention, the participant was required to meet a minimum criterion level of $80 \%$ accuracy at each level containing 20 stimuli.

The stimuli words were selected each week from one of four storybook narratives that were read to the child during the first session of each week. This type of narrative platform was used to increase the participant's attention toward print and to provide a more authentic context for intervention. McFadden (1998) suggested that teaching phonological awareness within the context of meaningful text provides positive benefits to the child due to the reciprocal nature of phonological awareness and literacy. Four levels of phonological awareness intervention included: (1) phoneme identification tasks, in which the child was taught to (a) listen to the word and (b) identify the first, last, or middle sound verbally; (2) phoneme blending tasks, in which the child was taught to (a) listen to individual phonemes presented in one-second intervals and (b) blend the phonemes in order to produce the targeted word; (3) phoneme segmentation tasks, in which the child was taught to analyze the stimuli words by (a) counting the number of sounds in the word and then (b) producing the individual sounds in each stimulus word; and (4) manipulation tasks in which the child was taught to delete and/or substitute phonemes in the targeted word to form a new word (see Appendix).

Traditional language intervention - The language intervention utilized a narrative (storybook) platform and focused on story retell and syntactic skills. In addition to their weakness in morphosyntactic processing, children with SLI are also known to have difficulties producing age-appropriate narratives (Swanson, Fey, Mills, \& Hood, 2005). The primary goal of the narrative intervention was for the child to retell a complete episode with all of the story grammar elements present (e.g., character, setting, initiating event, feeling, planned attempts to resolve the stated problem, and results/consequences). Each week of treatment, a specific book (e.g., The Fox and the Crow (Percy, 2000)) was selected for the narrative-based intervention in accordance with a particular theme.

The Story Grammar Marker (SGM; Moreau-Rooney \& Fidrych, 2002) was used for the narrative retell intervention. The SGM is an icon based intervention tool designed to facilitate understanding and development of narrative structure. The clinician read the book in its entirety and then immediately modeled the narrative retell using the SGM as the visual support. The child then retold the narrative using visual supports with the clinician scaffolding (e.g., modeling, questioning, cloze procedure) as needed. The syntax goals targeted included conjunctions, elaborated noun phrases, verb phrase elaboration, and sentence adverbs. During the story retell, the targeted syntactic goals were recast when an error was made by the child (Weismer \& Robertson, 2006). 


\subsection{Treatment fidelity}

For the purpose of monitoring the quality and consistency of the intervention, the trained graduate student clinicians participated in a five-hour instructional session on the implementation of both the phonological awareness intervention and traditional language intervention and were provided with a detailed script of the intervention prior to the initiation of the study. The intervention sessions were supervised by one of three certified speech-language pathologists for a minimum of $30 \%$ of the time. Following each supervised session, an evaluation checklist was completed and the result was discussed with the graduate clinician regarding the execution and adherence to the intervention protocol. Additionally, the second author of the study observed each of the graduate clinician's implementation of the phonological awareness intervention for a minimum of three sessions during the study in order to further ensure the quality and fidelity of the research. The supervisors assessed the fidelity of the phonological awareness intervention, using a 5-point Likert scale (poor to exemplary). Ratings of the implementation quality were: phoneme identification/ blending/segmentation tasks $(M=5.8, S D$ $=.75)$ and phoneme manipulation tasks $(M=4.7, S D=.57)$, and the overall phonological awareness intervention program $(M=4.8, S D=.58)$. Any discrepancy in adherence to the intervention protocol was discussed and feedback was given.

\subsection{Interrater reliability}

To determine interrater reliability for scoring pre- and post-testing data, two trained reliability coders, who were graduate students, recalculated raw and standardized scores for 25 randomly selected participants (50\% of the entire sample) while remaining blind to the initial test scores. Then, they checked their scores against the original scores in the Microsoft Office Excel spreadsheet. The coders and the test administrator were blind to each other's scoring. The two coders and the test administrator conducted an item-by-item comparison of their respective responses to each item administered in the battery. A reliability score was calculated for all variables by dividing the number of agreements by the number of disagreements plus agreements and multiplying by 100 . Interrater agreement was $95.2 \%$ agreement for coding raw data and $99 \%$ agreement for converting raw scores to standard scores.

\section{Results}

\subsection{Statistical considerations}

First, in order to establish whether our phonological awareness intervention program improved word-level reading skills of children in the PA-Tx group, two separate mixed-model analyses of variance (ANOVAs) were conducted on the Word Identification and Word Attack Next, pre- to post-test gains in the verbal memory tests were evaluated using a 2 × 2 mixed-model multivariate analyses of variance (MANOVA). In both analyses, group membership (PA-Tx vs. LI-Tx) served as the between-subjects factor and pre- and post-test scores served as the within-subjects factor. When a significant difference was found in the MANOVA, a series of univariate mixed-model ANOVAs and corresponding paired sample $t$ tests were conducted using a Bonferroni corrected alpha level in order to reveal the sources of multivariate significance and efficacy of intervention. Effect sizes of statistically significant results in the mixed-model ANOVAs are reported using the partial eta squared statistic $\left(\eta_{\rho}^{2}\right)$. To quantify the magnitude of change in mean pre- and post-test scores, effect sizes are reported using Cohen's d, for which 0.2 is small, 0.5 is medium, and 0.8 is large (Cohen, 1988).

\subsection{Gains on outcome measures}

Table 2 presents the means, standard deviations, post hoc comparisons for all outcome measures at both preand post-test intervals. 
Park, J., Ritter, M., Lombardino, L., Wiseheart, R., \& Sherman, S.

\section{Table 2}

Pre- and post-intervention group means and standard deviations with the results of paired tests for word-level reading and verbal memory measures

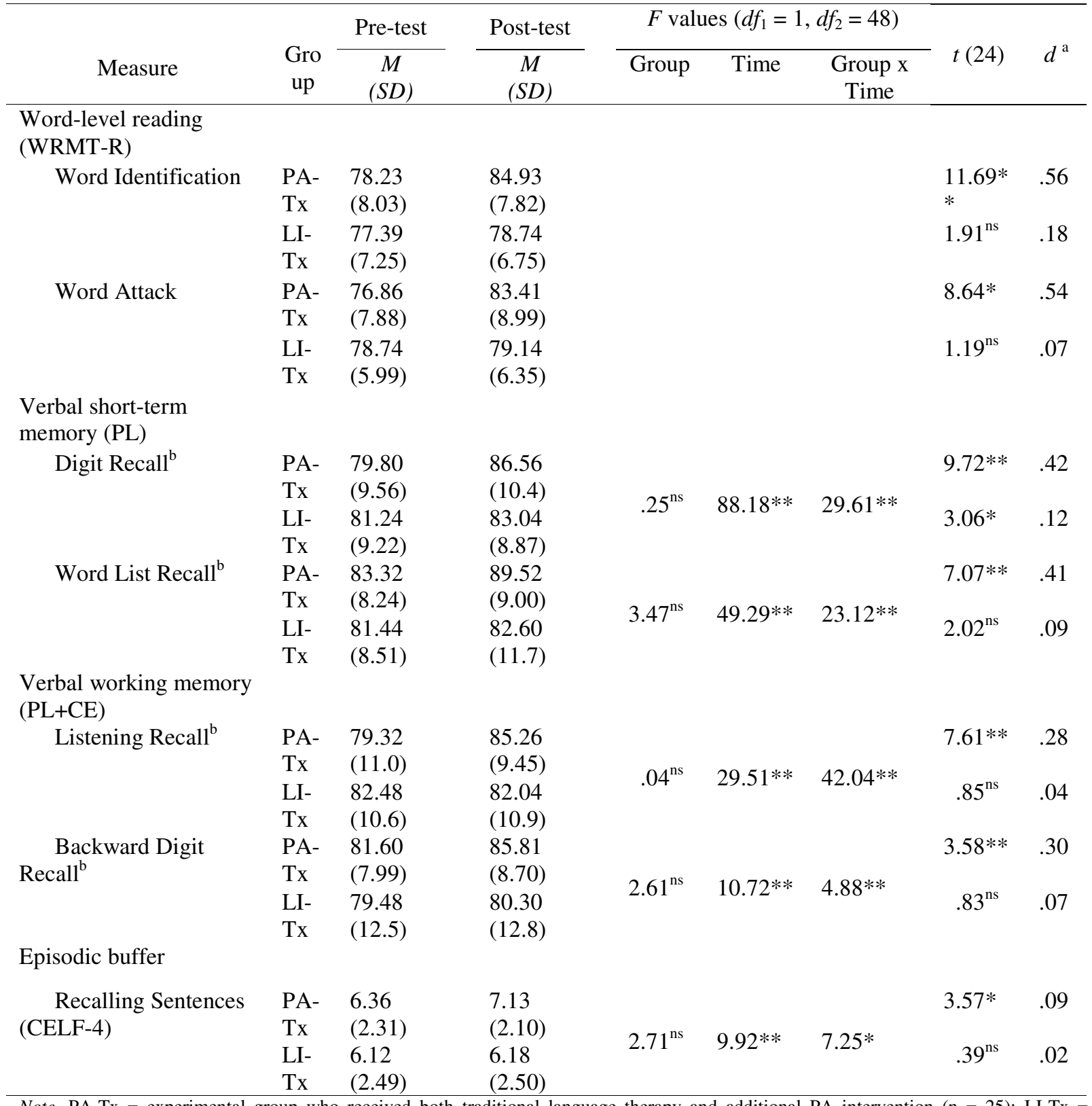

Note. PA-Tx $=$ experimental group who received both traditional language therapy and additional PA intervention $(\mathrm{n}=25)$; LI-Tx $=$ age-matched control group who received only traditional language therapy $(n=25)$; TONI-4 = Test of Nonverbal Intelligence -4 th Edition (TONI-4; Brown, Sherbenou, \& Johnsen, 2010); CELF-4 CLS = Core Language Scores of the Clinical Evaluation of Language Fundamentals-4 (Semel et al., 2003); WRMT-R = Woodcock Reading Mastery Test-Revised (Woodcock, 1988).

${ }^{\mathrm{a}}$ Group equivalence on gender was tested by chi-square analysis $[\chi 2(1, \mathrm{n}=50)=.725, p=.395]$. ${ }^{\mathrm{b}}$ Subtests of the Working Memory Test Battery for Children (WMTB-C; Pickering \& Gathercole, 2001).

${ }^{*} p<.01,{ }^{* *} p<.001$

Word decoding measures - For the Word Identification scores, the main effects for Time, $F(1,48)=78.359$, $p<.001, \eta_{\rho}^{2}=.620$, Group, $F(1,48)=11.19, p<.05, \eta_{\rho}^{2}=.113$, and Group $\mathrm{x}$ Time interaction were all significant, $F(1,48)=34.674, p<.001, \eta_{\rho}^{2}=.419$. On the Word Attack scores, significant main effects were found for Time, $F(1,48)=69.463, p<.001, \eta_{\rho}{ }^{2}=.591$, and Group x Time interaction, $F(1,48)=53.662, p<.001$, $\eta_{\rho}{ }^{2}=.528$. However, the main effect of Group was not significant, $F(1,48)=.226, p=.636, \eta_{\rho}^{2}=.005$. Post hoc paired $t$ tests revealed that the Word Identification scores at post-test were significantly greater than pre-test for the PA-Tx group, $t(24)=11.69, p<.001, d=.56$ while no such difference was found for the LI-Tx group. 
Similarly, a significant pre- to post-test improvement was found for the PA-Tx group, $t(24)=8.64, p<.001, d$ $=.54$ while the LI-Tx group did not show a significant gain. Thus, word reading skills were significantly improved in children who received additional phonological awareness intervention with medium effect sizes. This significant training effect of phonological awareness intervention on word reading skills of school-age children with language impairment is consistent with previous intervention research.

\section{Verbal short-term memory}
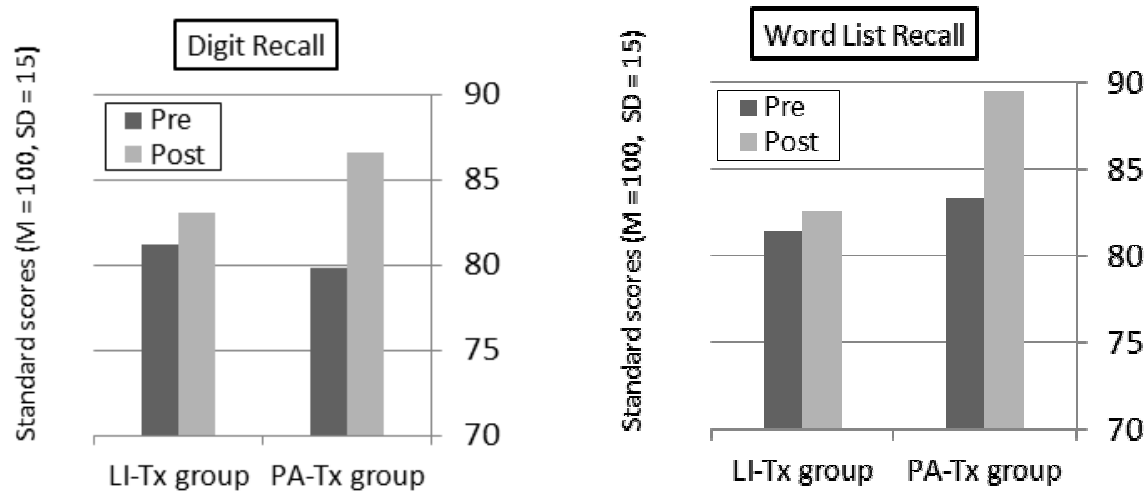

2. Verbal working memory
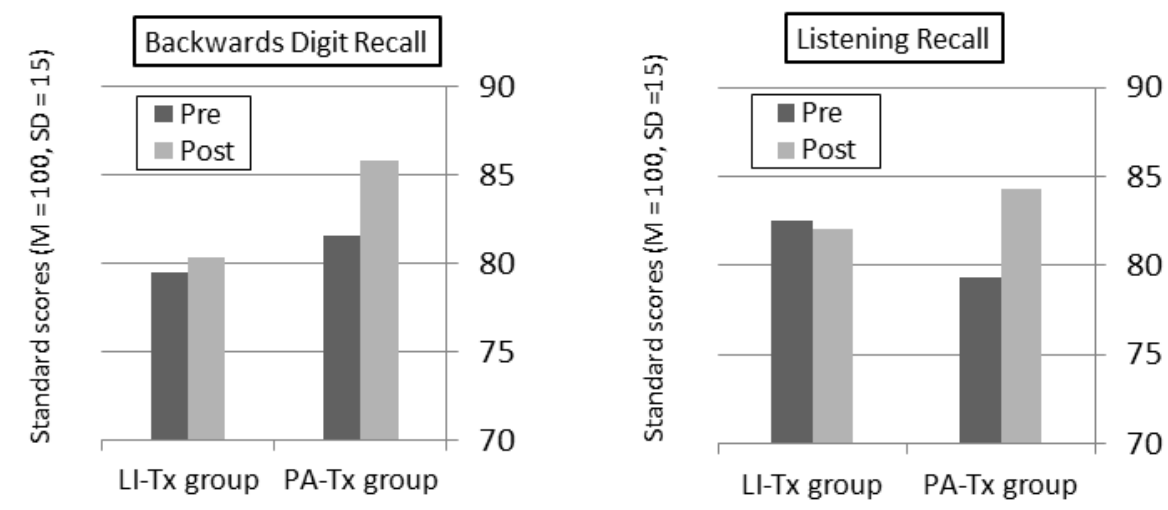

3. Episodic buffer

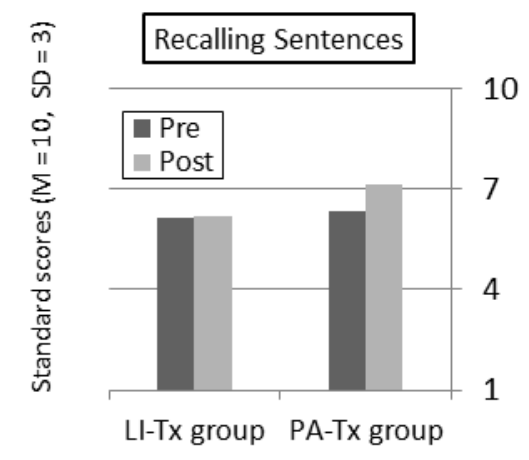

Figure 1. Group improvement on the verbal memory measures

Verbal memory measures - Figure 1 displays the two groups' pre- to post-test change in scores on the five verbal memory measures. Pre-intervention group means were remarkably similar across measures, the greatest group differences occurring on the Listening Recall subtest. Approximately $40 \%$ of participants in both groups (i.e., $41 \%$ for the PA-Tx and 37\% for the LI-Tx group, respectively) performed at least 1 SD below the normative mean on at least four of these five verbal memory subtests. This finding lends support to Archibald 
and Gathercole's (2006) hypothesis that children with SLI are likely to show verbal working memory deficits in both phonological loop and central executive functions. The omnibus MANOVA (Wilk's $\lambda$ ) revealed a significant main effect for Time, $F(1,48)=140.949, p<.001, \eta_{\rho}{ }^{2}=.746$, and a significant Group x Time interaction effect, $F(1,48)=77.436, p<.001, \eta_{\rho}^{2}=.617$. No significance main effect for Group was found, $F(1,48)=3.062, p$ $=.087, \eta_{\rho}{ }^{2}=.06$. A series of follow-up univariate mixed-model ANOVAs were conducted for each of these variables, where the principal interest was in the Group x Time interaction effect. A pattern of significant Group $\mathrm{x}$ Time interaction effect was noted for all subtests: Digit Recall, $F(1,48)=29.61, p<.001, \eta_{\rho}{ }^{2}=.648$; Word List Recall, $F(1,48)=23.12, p=.002, \eta_{\rho}^{2}=.325$; Listening Recall, $F(1,48)=42.04, p<.001, \eta_{\rho}{ }^{2}=.467$; Backwards Digit Recall, $F(1,48)=4.88, p=.032, \eta_{\rho}{ }^{2}=.092$; and Recalling Sentences, $F(1,48)=7.25, p=.003$, $\eta_{\rho}{ }^{2}=.131$. Similarly, univariate mixed-model ANOVAs showed a significant Time main effect for all verbal memory measures. However, Group main effect was not significant for any measure.

In order to further analyze the source of significant Time and Group x Time main effects, multiple paired sample $t$ tests were conducted for the five verbal memory measures using a Bonferroni corrected $\alpha$ of 0.005 . First, post hoc analyses on the measures of the verbal short-term memory yielded mixed results. On the Word List Recall subtest, only the PA-Tx group showed significant improvement as evidenced by a small to medium effect size, $t(24)=7.07, p<.001, d=.41$ while no such significant gains were noted for the LI-Tx group, $t(24)=2.02$, $p=.054, d=.09$. By contrast, both groups showed significant pre-post gains in the Digit Recall subtest [PA-Tx, $t(24)=9.72, p<.001 ;$ LI-Tx, $t(24)=3.06, p=.005]$. It should be noted, however, that the gains made by the PA-Tx group were much greater than the gains made by the LI-Tx group for both tasks as evidenced by the effect sizes $\left(d_{\mathrm{PA}-\mathrm{Tx}}=.42, d_{\mathrm{LI}-\mathrm{Tx}}=.12\right)$.

Next, post hoc tests for the two subtests of the verbal working memory component revealed significant pre-post gains only for the PA-Tx group: Listening Recall, $t(24)=7.61, p<.001$ and Backwards Digit Recall, $t(24)=3.58, p=.002$. No such pre-post gains were noted for the LI-Tx group. For the PA-Tx group, effects sizes were small for both subtests $(d=.28-.30)$, suggesting that verbal working memory skills improved at a similar rate for both tasks. Last, only the PA-Tx group showed significant pre-post progress on the measure of the episodic buffer function (i.e., Recalling Sentences) with a fairly small effect size, $t(24)=3.57, p=.002, d=.09$. No significant pre-post differences were observed for the LI-Tx group (see Figure 1c). Overall, inspection of Figure 1 indicates that only children in the PA-Tx group consistently made significant progress across all verbal memory measures with the exception of one PL measure (i.e., digit recall), in which the control group also made significant progress with a small effect size.

\section{Discussion}

The aim of the current study was to investigate the effects of explicit phonological awareness intervention on a range of verbal memory measures among a subgroup of school-age children with SLI and concurrent difficulties with single word reading. To our best knowledge, this is the first study that indicates 'generalized' cross-component efficacy of phonological awareness intervention to all three aspects of Baddeley's verbal working memory.

We posed two research hypotheses. Our first hypothesis was that phonological awareness training would generalize to improvements in the three substrates of Baddeley's verbal working memory model (i.e., verbal short-term memory, verbal working memory, and episodic buffer) as a result of improving underlying phonological representations and shared operational mechanisms between phonological awareness and verbal working memory tasks (Baddeley, 2000, 2003). Our findings were surprisingly clear cut: school-age children with SLI receiving a phonological awareness intervention significantly outperformed the children in the control group on all verbal memory measures. These findings lead us to infer that phonological awareness training improves not only phonological representations typically observed in children with SLI but also verbal memory capacity more generally. 
Further, given the contrasting role of the three components of the verbal memory system, we also hypothesized that explicit phonological awareness intervention would result in differential gains in each of the three components. The strongest effects were found for the digit recall and word list recall subtests, which were used to assess the verbal short-term memory component of the model (i.e., phonological loop). Because these simple span tasks require relatively little attentional and organizational resources, we attribute the strong effects to the improved quality of the underlying phonological representations acquired during the phonological awareness intervention.

The next largest effect sizes were reported for the subtests used to measure the verbal working memory functioning (i.e., PL $+\mathrm{CE}$ ), for which the averaged effect size was small to medium. The finding may be related to the nature or level of the complexity of the verbal memory tasks. In other words, our selected verbal short-term memory subtests may be easier for children and more amenable to explicit phonological awareness intervention. As mentioned previously, verbal working memory tasks require the integration of processes (attention, organization) beyond rote memory. Thus, the relatively smaller gains found in the verbal working memory subtests used in the present study reflect in part the apparent gap in complexity between verbal complex span tasks and short-term memory tasks (Gathercole \& Pickering, 2000). This explanation is in line with the recent neuroanatomical fMRI studies conducted to explore the neural correlates of both forward digit recall (i.e., PL) and backward digit recall in healthy adults offering compelling evidence for activation of separate neural systems for verbal short-term memory and complex working memory tasks (Buchsbaum \& D’Esposito, 2008).

Finally, the smallest changes between pre- and posttest scores were found on the recalling sentences subtest, which was chosen to represent the episodic buffer component of the verbal working memory model. The episodic buffer serves as a limited temporary store system capable of integrating information from the multiple systems of working memory into a unitary episodic chunk. Efficient sentence recall requires the integration of semantic, syntactic, and morphological knowledge needed to structurally decompose sentences. Due to the multidimensional functions of the episodic buffer, it may be the least amenable component of the model to be influenced by highly explicit phonological awareness intervention. It is also well established that a sentence recall task heavily relies on the quality of prior language skills in long-term memory (Alloway \& Gathercole, 2005). Hence, the episodic buffer function in children with SLI might be more resistant to change than in nonclinical populations because their language processing deficits are a major clinical marker of SLI in both children and adults (Archibald \& Joanisse, 2009; Bishop \& Adams, 1990; Poll, Betz, \& Miller, 2010).

\section{Conclusions, implications, and limitations}

Due to the common operational mechanism between tasks of phonological awareness and verbal memory skills, phonological awareness intervention should increase our participants' verbal memory capacity. Verifying this assumption should be an important goal of the literature, as improved working memory capacity is the mechanism through which major cognitive activities occur such as word decoding, reading comprehension, writing, and high-level textual inference.

We found clear cut generalization effects of phonological awareness intervention on a range of verbal memory skills. Our data show that children with SLI in second through third grade can make significant gains on both directly targeted skills (i.e., word decoding) and untrained verbal working memory skills. Also, our findings suggest that phonological awareness intervention differentially affected the three subcomponents of Baddeley's $(2000,2003)$ verbal working memory model. Of note, effect sizes associated with pre-post intervention changes align with the complexity of functions associated with each component of the model: the strongest gains were found on tasks most dependent on phonological representations and the weakest gains were found on tasks less dependent on phonological representations and more dependent on cognitive skills associated with attention, integration, and long-term memory.

Aside from our theoretical speculations, our results have far reaching practical implications for phonological 
Park, J., Ritter, M., Lombardino, L., Wiseheart, R., \& Sherman, S.

awareness intervention with school-age children with SLI and concomitant word-reading difficulties. First, perhaps the most important finding of the current study was that improvements in verbal memory abilities were accomplished in a relatively short period of time (i.e., four 20-min weekly sessions for a total of 4 weeks). This is an important finding considering that it is typical that phonological awareness intervention programs are implemented over a long period of time in intensive settings (Gillon, 2004). Second, the present study underscores the importance of phonological awareness intervention beyond first grade as a viable option for improving both word decoding and verbal memory skills. We interpret this finding as evidence that phonological awareness intervention training exerts qualitative changes on underlying phonological representations which, in turn, make verbal memory processes more efficient. Many practitioners observe that students beyond first grade still show difficulty with reading and spelling as they cannot make the conscious connection between sound and print due to their lack of phonological awareness.

To achieve successful scholastic progress, children in upper elementary grades who are struggling with reading may benefit from explicit instruction that will enhance their phonological awareness. Unfortunately, underdeveloped phonological awareness skills noted in these struggling readers are often overlooked in the classroom since reading intervention and assessment for these students in upper grades generally focus on text comprehension and not awareness of phonological units (Denton, Bryan, Wexler, Reed, \& Vaughn, 2007). Teachers and speech-language pathologists can develop and implement direct phonological awareness training integrated within regular classroom activities and developed to fit with the classroom's regular reading program. For example, in a storybook-based phonological awareness intervention, phonological awareness skills can be taught in the context of familiar stories and experiences (Justice and Ezell, 2002).

The generalizability of the current study is limited by its sample size of only 25 subjects per group. Future studies will be needed to confirm the training effects with a larger sample size and to address additional questions about the mechanisms underlying training-induced improvements in verbal memory skills. Furthermore, no other language measures were collected throughout the intervention period and thus carryover of the gains made during the intervention period to other language domains could not be assessed. Third, the current study was short-term, thus we do not know whether improvements in verbal working memory will be maintained long-term. Follow-up data are needed to determine the long-term stability of these intervention effects.

\section{References}

Alloway, T. P., \& Gathercole, S.E. (2005). The role of sentence recall in reading and language skills of children with learning difficulties. Learning and Individual Differences, 15, 271-282. http://dx.doi.org/10.1016/j.lindif.2005.05.001

Archibald, L. M. D., \& Gathercole, S. E. (2006). Non-word repetition: A comparison of tests. Journal of Speech, Language, and Hearing Research, 49, 970-983. http://dx.doi.org/10.1044/1092-4388(2006/070)

Archibald, L. M., \& Gathercole, S. E. (2006). Short-term and working memory in specific language impairment. In T. P. Alloway \& S. E. Gathercole (Eds.), Working memory in neurodevelopmental conditions (pp. 139-160). Hove, England: Psychology Press.

Archibald, L. M., \& Joanisse, M. F. (2009). On the sensitivity and specificity of nonword repetition and sentence recall to language and memory impairments in children. Journal of Speech, Language, and Hearing Research, 52, 899-914. http://dx.doi.org/10.1044/1092-4388(2009/08-0099)

Baddeley, A. D. (1986). Working memory. Oxford, England: Oxford University Press.

Baddeley, A. D. (2000). The episodic buffer: A new component of working memory? Trends in Cognitive Sciences, 4, 417-423. http://dx.doi.org/10.1016/S1364-6613(00)01538-2

Baddeley, A. D. (2003) Working memory and language: An overview. Journal of Communication Disorders, 36 , 189-208. http://dx.doi.org/10.1016/S0021-9924(03)00019-4

Baddeley, A. D., \& Wilson, B. (2002). Prose recall and amnesia: Implications for the structure of working memory. Neuropsychologia, 40, 1737-1743. http://dx.doi.org/10.1016/S0028-3932(01)00146-4 
Phonological awareness intervention for verbal working memory skills in school-age children

Baddeley, A. D., \& Hitch, G. (1974).Working memory. In G. A. Bower (Ed.), Recent advances in learning and motivation (Vol. 8, pp. 47-90). New York, NY: Academic Press.

Bishop, D. V. M., \& Snowling, M. J. (2004). Developmental dyslexia and specific language impairment: Same or different? Psychological Bulletin, 130, 858-886. http://dx.doi.org/10.1037/0033-2909.130.6.858

Bishop, D.V.M., \& Adams, C. (1990). A prospective study of the relationship between specific language impairment, phonological disorders and reading retardation. Journal of Child Psychology and Psychiatry, 31, 1027-1050. http://dx.doi.org/10.1111/j.1469-7610.1990.tb00844.x

Brown, L., Sherbenou, R. J., \& Johnsen, S. K. (2010). Test of nonverbal intelligence (4th ed.). Austin, TX: PRO-ED.

Buchsbaum, B. R., \& D'Esposito, M. (2008). The search for the phonological store: From loop to convolution. Journal of Cognitive Neuroscience, 20, 762-778. http://dx.doi.org/10.1162/jocn.2008.20501

Castles, A., \& Coltheart, M. (2004). Is there a causal link from phonological awareness to success in learning to read? Cognition, 91, 77-111. http://dx.doi.org/10.1016/S0010-0277(03)00164-1

Catts, H. W., Adlof, S. M., Hogan, T. P., \& Weismer, S. (2005). Are specific language impairment and dyslexia distinct disorders? Journal of Speech, Language, and Hearing Research, 48, 1378-1396. http://dx.doi.org/10.1044/1092-4388(2005/096)

Catts, H., Bridges, M., Little, T., \& Tomblin, J.B. (2008). Reading achievement growth in children with language impairments. Journal of Speech, Language, and Hearing Research, 51, 722-738. http://dx.doi.org/10.1044/1092-4388(2008/07-0259)

Catts, H., Fey, M, Tomblin, J., \& Zhang, X. (2002). Longitudinal investigation of reading outcomes in children with language impairment. Journal of Speech and Hearing Research, 45, 1142-1157. http://dx.doi.org/10.1044/1092-4388(2002/093)

Catts, H., Fey, M., Zhang, X., \& Tomblin, B. (2001). Estimating the risk of future reading difficulties in kindergarten children: A research-based model and its clinical implementation. Language, Speech, and Hearing Services in Schools, 32, 32-50.http://dx.doi.org/10.1044/0161-1461(2001/004)

Chou, C. P., Wang, S., \& Ching, G. S. (2012). Balanced reading instructions: An action research on elementary cram school students. International Journal of Research Studies in Language Learning, 1, 3-20. http://dx.doi.org/10.5861/ijrsll.2012.v1i1.7

Cohen, J. (1988). Statistical power analysis for the behavioral sciences $\left(2^{\text {nd }}\right.$ ed.). Hillsdale, NJ: Erlbaum.

Conway, A. R. A., Kane, M. J., \& Engle, R. W. (2003). Working memory capacity and its relation to general intelligence. Trends in Cognitive Sciences, 7, 547-552. http://dx.doi.org/10.1016/j.tics.2003.10.005

Cunningham, A. E. (1990). Explicit versus implicit instruction in phonemic awareness. Journal of Experimental Child Psychology, 50, 429-444. http://dx.doi.org/10.1016/0022-0965(90)90079-N

De Jong, P. F., Seveke, M. J., \& van Veen, M. (2000). Phonological sensitivity and the acquisition of new words in children. Journal of Experimental Child Psychology, 76, 275-301. http://dx.doi.org/10.1006/jecp.1999.2549

Denton, C., Bryan D., Wexler, J., Reed, D., \& Vaughn, S. (2007). Effective instruction for middle school students with reading difficulties: The reading teacher's sourcebook. Texas: Texas Education Agency

Dollaghan, C., \& Campbell, T. F. (1998). Nonword repetition and child language impairment. Journal of Speech, Language, and Hearing Research, 41, $1136-1146$.

Fowler, A. (1991). How early phonological development might set the stage for phoneme awareness. In S. Brady \& D. Shankweiler (Eds.), Phonological processes in literacy: A tribute to Isabelle Y. Liberman (pp. 97-118). Hillsdale, NJ: Erlbaum.

Garlock, V. M., Walley, A. C., \& Metsala, J. L. (2001). Age-of-acquisition, word frequency and neighbourhood density effects on the development of spoken word recognition and phoneme awareness. Journal of Memory and Language, 45, 468-492. http://dx.doi.org/10.1006/jmla.2000.2784

Gathercole, S. E., \& Baddeley, A. D. (1990). Phonological memory deficits in language disordered children: Is there a causal connection? Journal of Memory and Language, 29, 336-360. http://dx.doi.org/10.1016/0749-596X(90)90004-J

Gathercole, S. E., \& Pickering, S. J. (2001). Working memory deficits in children with special educational needs. 
Park, J., Ritter, M., Lombardino, L., Wiseheart, R., \& Sherman, S.

British Journal of Special Education, 28, 89-97. http://dx.doi.org/10.1111/1467-8527.00225

Gillam, R., \& van Kleeck, A. (1996). Phonological awareness training and short-term memory: Clinical implications. Topics in Language Disorders, 17, 72-82.

http://dx.doi.org/10.1097/00011363-199611000-00008

Gillon, G. (2004). Phonological awareness: From research to practice. New York: Guilford Press.

Goldsworthy, C. (2004). Developmental reading disabilities: A language based treatment approach. San Diego, CA: Singular Publishing Group.

Griffith, P. L., \& Olson, M.W. (1992). Phonemic awareness helps beginning readers break the code. The Reading Teacher, 45, 516-523.

Halpern, C., McMillan, C., Moore, P., Dennis, K., \& Grossman, M. (2003). Calculation impairment in focal neurodegenerative diseases. Journal of Neurological Sciences, 208, 31-38. http://dx.doi.org/10.1016/S0022-510X(02)00416-1

Henry, L. A., \& Millar, S. (1993). Why does memory span improve with age? A review of the evidence for two current hypotheses. European Journal of Cognitive Psychology, 5, 241-287. http://dx.doi.org/10.1080/09541449308520119

Hurford, D. P., \& Sanders, R. E. (1990). Assessment and remediation of a phonemic discrimination deficit in reading disabled second- and fourth-graders. Journal of Experimental Child Psychology, 50, 396-415. http://dx.doi.org/10.1016/0022-0965(90)90077-L

Justice, L. M., \& Ezell, H. K. (2000). Enhancing children's print and word awareness through home-based parent intervention. American Journal of Speech-Language Pathology, 9, 257-269.

Kane, M. J., Brown, L. H., Little, J. C., Silvia, P. J., Myin-Germeys, I., \& Kwapil, T. R. (2007). For whom the mind wanders and when: An experience-sampling study of working memory and executive control in daily life. Psychological Science, 18, 614-621. http://dx.doi.org/10.1111/j.1467-9280.2007.01948.x

King, J., \& Just, M. A. (1991). Individual differences in syntactic processing: The role of working memory. Journal of Memory and Language, 30, 580-602. http://dx.doi.org/10.1016/0749-596X(91)90027-H

Kovelman, I., Norton E. S., Gaab, N., Christodoulou J. A., Triantafyllou, C., Lieberman D. A., Lymberis, J., Witfield-Gabrieli, S., Wolf, M., \& Gabrieli J. D. E.(2012). Brain bases of phonological awareness for spoken language in children and its dysfunction in dyslexia. Cerebral Cortex, 22, 745-764. http://dx.doi.org/10.1093/cercor/bhr094

Leather, C. V., \& Henry, L. A. (1994). Working memory span and phonological awareness tasks as predictors of early reading ability. Journal of Experimental Child Psychology, 58, 88-111. http://dx.doi.org/10.1006/jecp.1994.1027

Leonard, L. B. (1998). Children with specific language impairment. Cambridge, MA: MIT Press.

Lundberg, I., Frost, J., \& Petersen, O. (1988). Effects of an extensive program for stimulating phonological awareness in preschool children. Reading Research Quarterly, 23, 263-284.

http://dx.doi.org/10.1598/RRQ.23.3.1

Lyon, G. R., Shaywitz, S.E., \& Shaywitz, B.A. (2003). A definition of dyslexia. Annals of Dyslexia, 53, 1-15. http://dx.doi.org/10.1007/s11881-003-0001-9

McArthur, G. M., Hogben, J. H., Edwards, V. T., Heath, S. M., \& Mengler, E. D. (2000). On the "specifics" of specific reading disability and specific language impairment. Journal of Child Psychology and Psychiatry, 41, 869 - 874. http://dx.doi.org/10.1111/1469-7610.00674

McFadden, T. U. (1998). Sounds and stories: Teaching phonemic awareness in print contexts. American Journal of Speech-Language Pathology, 7, 5-13.

Melby-Lervåg, M., \& Hulme, C. (2010). Serial and free recall in children can be improved by training: Evidence for the importance of phonological and semantic representations in immediate memory tasks.

Psychological Science, 21, 1694-1700. http://dx.doi.org/10.1177/0956797610385355

Montgomery, J. W. (2000). Relation of working memory to offline and real-time sentence processing in children with specific language impairment. Applied Psycholinguistics, 21, 117-148. http://dx.doi.org/10.1017/S0142716400001065

Montgomery, J. W., \& Evans, J. L. (2009). Complex sentence comprehension and working memory in children 
Phonological awareness intervention for verbal working memory skills in school-age children

with specific language impairment. Journal of Speech, Language, and Hearing Research, 52, 269-288. http://dx.doi.org/10.1044/1092-4388(2008/07-0116)

Moreau-Rooney, M., \& Fidrych, H. (2002). Story grammar marker [Learning tool]. Springfield, MA: MindWing Concepts.

Navarro, J. I., Aguilar, M., Alcalde, C., Ruiz, G., Marchena, E., Menacho, I. (2011). Inhibitory processes, working memory, phonological awareness, naming speed, and early arithmetic achievement. The Spanish Journal of Psychology, 14, 580-588.http://dx.doi.org/10.5209/rev_SJOP.2011.v14.n2.6

Oakhill, J., \& Kyle, F. (2000). The relation between phonological awareness and working memory. Journal of Experimental Child Psychology, 75, 152-164. http://dx.doi.org/10.1006/jecp.1999.2529

Pennington, B. F., \& Bishop, D. V. (2009). Relations among speech, language, and reading disorders. Annual Review of Psychology.60, 283-306. http://dx.doi.org/10.1146/annurev.psych.60.110707.163548

Percy, G. (2000). Children's favorite animal fables. Barnes and Nobles.

Pickering, S. J., \& Gathercole, S. (2001). Working memory test battery for children. Minneapolis, MN: PsychCorp/Pearson.

Poll, G., Betz, S., \& Miller, C. (2010). Identification of clinical markers of specific language impairment in adults. Journal of Speech, Language, and Hearing Research, 53, 414-429. http://dx.doi.org/10.1044/1092-4388(2009/08-0016)

Roodenrys, S., \& Stokes J. (2001) Serial recall and nonword repetition in reading disabled children. Reading and Writing, 14, 379-394.http://dx.doi.org/10.1023/A:1011123406884

Roodenrys, S., Hulme, C., \& Brown, G. (1993). The development of short-term memory span: Separable effects of speech rate and long-term memory. Journal of Experimental Child Psychology, 56, 431-442. http://dx.doi.org/10.1006/jecp.1993.1043

Rvachew, S., Nowak, M., \& Cloutier, G. (2004). Effect of phonemic perception training on the speech production and phonological awareness skills of children with expressive phonological delay. American Journal of Speech-Language Pathology, 13, 250-263. http://dx.doi.org/10.1044/1058-0360(2004/026)

Schneider, W., Küspert, P., Roth, E., Vise, M., \& Marx, H. (1997). Short- and long-term effects of training phonological awareness in kindergarten: Evidence from two German studies. Journal of Experimental Child Psychology, 66, 311-340. http://dx.doi.org/10.1006/jecp.1997.2384

Schuele, C., \& Boudreau, D. (2008). Phonological awareness intervention: Beyond the basics. Language, Speech, and Hearing Services in Schools, 39, 3-20. http://dx.doi.org/10.1044/0161-1461(2008/002)

Semel, E., Wiig, E. H., \& Secord, V.A. (2003). Clinical Evaluation of Language Fundamentals-Fourth Edition. San Antonio, TX. The Psychological Corporation.

Shankweiler, D. (1989). How problems of comprehension are related to difficulties in decoding. In D. Schankweiler \& I. Y. Liberman (Eds.), Phonology and reading disability: Solving the reading puzzle (pp. 35-68). Research Monograph Series. Ann Arbor, MI: University of Michigan Press.

Shaywitz, S. E., \& Shaywitz, B. A. (2005). Dyslexia (specific reading disability). Biological Psychiatry, 57, 1301-1309. http://dx.doi.org/10.1016/j.biopsych.2005.01.043

Siegel, L. S., \& Linder, B. A. (1984). Short term memory processes in children with reading and arithmetic learning disabilities. Developmental Psychology, 20, 200-207. http://dx.doi.org/10.1037/0012-1649.20.2.200

Snowling, M., Bishop, D. V., \& Stothard, S. E. (2000). Is preschool language impairment a risk factor for dyslexia in adolescence? Journal of Child Psychology and Psychiatry, 41, 587- 600. http://dx.doi.org/10.1111/1469-7610.00651

Stanovich, K. E., Cunningham, A. E., \& Freeman, D. J. (1984). Intelligence, cognitive skills and early reading progress. Reading Research Quarterly, 19, 278-303. http://dx.doi.org/10.2307/747822

Swanson, H. L., \& Berninger, V. W. (1996). Individual differences in children's working memory and writing skills. Journal of Experimental Child Psychology, 63, 358-385. http://dx.doi.org/10.1006/jecp.1996.0054

Swanson, H. L., \& Ashbaker, M. H. (2000). Working memory, short-term memory, speech rate, word recognition, and reading comprehension in learning disabled readers: Does the executive system have a role? 
Park, J., Ritter, M., Lombardino, L., Wiseheart, R., \& Sherman, S.

Intelligence, 28, 1-30. http://dx.doi.org/10.1016/S0160-2896(99)00025-2

Swanson, L. A., Fey, M. E., Mills, C. E., \& Hood, L. S. (2005). Use of narrative-based language intervention with children who have specific language impairment. American Journal of Speech-Language Pathology, 14, 131-143. http://dx.doi.org/10.1044/1058-0360(2005/014)

Troia, G. A., Roth, F. P., \& Yeni-Komshian, G. H. (1996). The relationship between word retrieval and phonological awareness in normally-developing children. Journal of Speech and Hearing Research, 39, 1099-1108.

van Kleeck, A., Gillam, R. B., \& Hoffman, L. M. (2006). Training in phonological awareness generalizes to phonological working memory: A preliminary investigation. The Journal of Speech-Language Pathology and Applied Behavior Analysis, 1, 228 - 243.

Vitale, M. R., \& Romance, N. R. (2012). A research-based strategy for inductively accelerating vocabulary acquisition of at-risk students in Grade 4. International Journal of Research Studies in Language Learning, 1, 33-46. http://dx.doi.org/10.5861/ijrsll.2012.v1i2.73

Weismer, S. E., \& Robertson, S. (2006). Focused stimulation approach to language intervention. In R. McCauley $\&$ M. Fey (Eds.), Treatment of language disorders in children. Baltimore: Paul H. Brookes.

Weismer, S. E., \& Edwards, J. (2006). The role of phonological storage deficits in specific language impairment: A reconsideration. Invited commentary on S.E. Gathercole, Nonword repetition and word learning: The nature of the relationship. Applied Psycholinguistics, 27, 556-562. http://dx.doi.org/10.1017/S0142716406240396

Woodcock, R. W. (1988). Woodcock reading mastery test-revised. Circle Pines, MN: American Guidance Service.

Appendix: An abridged example of phonological awareness intervention tasks for The Fox and the Crow (Percy, 2000).

Materials: (a) Two sets of 10 stimuli (e.g., 20 different words selected from The Fox and the Crow or words that are representative of words found in the text). (b) Eight Colored Blocks representing individual sounds in words.

Phoneme Identification: Identify sounds in the initial and final position of words.

Clinician: "Tell me the first sound in the word .$"$

CVC

fine

had

Clinician: "Tell me the last sound in the word

$\underline{\text { CVC }}$

his

fine
Correct Response

/f/

/h/

\section{Correct Response}

/z/

/n/ 
Phonological awareness intervention for verbal working memory skills in school-age children Phoneme Blending: Progress from blending CV/VC combinations to blending CCVCC combinations.

Clinician: "After I say the sounds, put the sounds together to create a new word."

\section{$\underline{\mathrm{CV} \text { and } \mathrm{VC}}$}

i-n

$\mathrm{i}-\mathrm{t}$

$\underline{\text { CVC }}$

ch-ee-se

f-i-ne

\section{$\underline{\text { CCVC }}$}

g-1-ea-m

b-r-ea-d

$\underline{\mathrm{CCVCC}}$

g-r-a-n-d

s-n-i-ff-t

\section{$\underline{\text { Correct Response }}$}

in

it

\section{Correct Response}

cheese

fine

\section{Correct Response}

gleam

bread

\section{Correct Response}

grand

sniffed

Phoneme segmentation: Segmenting words into individual phonemes progresses from monosyllabic words to multi-syllabic words.

Clinician: "Tell me the sounds that you hear in this word."

$\underline{\mathrm{CV}}$ and VC

in

he

$\underline{\mathrm{CVC}}$

his

that

$\underline{\mathrm{CCVC}}$

small

sweet

\section{Correct Response (sounds)}

i-n (2)

h-e (2)

Correct Response (sounds)

h-i-s (3)

th-a-t (3)

$\underline{\text { Correct Response (sounds) }}$

s-m-a-11 (3)

s-w-ee-t (3) 
Park, J., Ritter, M., Lombardino, L., Wiseheart, R., \& Sherman, S.

\section{$\underline{\mathrm{CCVCC}}$}

grand

crept

$\underline{\text { Multisyllabic Words }}$

watching

strutted
Correct Response (sounds)

$$
\begin{aligned}
& \text { g-r-a-n-d (5) } \\
& \text { c-r-e-p-t (5) }
\end{aligned}
$$

\section{$\underline{\text { Correct Response (sounds) }}$}

$$
\begin{aligned}
& \text { w-a-tch-i-ng (5) } \\
& \text { s-t-r-u-tt-e-d (7) }
\end{aligned}
$$

Phoneme manipulation/deletion. Deleting sounds in words to form new words or nonwords.

Clinician: "Say , now say without

$\underline{\mathrm{CVC}}$

sack without /s/

cheese without /z/

\section{Correct Response}

ack

chee

Phoneme manipulation/replacement. Replacing sounds in words to form new words or nonwords. Progresses from initial word position to final and medial word positions.

Clinician: "Say , now say instead of

\section{CVC: Initial Replacement}

make: say $/ \mathrm{t} /$ instead of the $/ \mathrm{m} /$ sound

bowl: say /k/ instead of the /b/ sound

CVC: Final Replacement

make: say /t/ instead of the $/ \mathrm{k} /$ sound

base: say /t/ instead of the /s/ sound

CVC/CCV: Medial Replacement

crow: say /e/ instead of the /o/ sound

make: say /i/ instead of the /e/ sound

\section{$\underline{\text { Correct Response }}$}

take

coal

\section{Correct Response}

mate

bait

\section{Correct Response}

cray

meek 